\title{
Accuracy Improvement Calibrations for the Double-Position 4-PPPS Aircraft Docking System
}

\author{
Ruolong Qi $\mathbb{D}^{1}{ }^{1}$ Yuangui Tang $\mathbb{D}^{2}{ }^{2}$ and Ke Zhang $\mathbb{D}^{1}$ \\ ${ }^{1}$ School of Mechanical Engineering, Shenyang Jianzhu University, Shenyang, Liaoning, China \\ ${ }^{2}$ The State Key Laboratory of Robotics, Shenyang Institute of Automation, Chinese Academy of Sciences, \\ Shenyang, Liaoning, China \\ Correspondence should be addressed to Ruolong Qi; qiruolong@126.com
}

Received 3 February 2020; Revised 30 March 2020; Accepted 7 April 2020; Published 25 April 2020

Academic Editor: Francesco Aggogeri

Copyright (C) 2020 Ruolong Qi et al. This is an open access article distributed under the Creative Commons Attribution License, which permits unrestricted use, distribution, and reproduction in any medium, provided the original work is properly cited.

\begin{abstract}
A double-positions 4-PPPS parallel mechanism is used for the aircraft fuselage assembly process to improve the docking efficiency and reduce the labor intensity. However, the accuracy is hard to guarantee, for the mechanism is large and redundant and has manufacturing and assembly errors. To improve the accuracy of the 4-PPPS parallel aircraft fuselage docking system, firstly, an averaging iteration method is proposed to calibrate the datum points in the airplane coordinate which are the references of the entire docking system. And secondly, a kinematic calibration method based on the derivative of the spatial pose transformation is proposed to calibrate up to 42 kinematic parameters. By these two methods, the final maximum position error reduced from $2.2 \mathrm{~mm}$ to $0.035 \mathrm{~mm}$ and the maximum pointing error reduced from 0.08 degree to 0.018 degree. The accuracy measurement and docking experiment prove the efficiency of the proposed methods.
\end{abstract}

\section{Introduction}

Fuselages of the airplane are large deformable parts; it is very hard to adjust their postures or assemble them together accurately. Airplane docking systems are designed to promote the accuracy and efficiency of the assembly process. The principle of the docking system is that two mechanisms carry two fuselages, respectively, and join them together accurately. It means that the accuracy of the docking system determines the results of fuselage docking. A fuselage docking system designed for MA700 airplane is shown in Figure 1. There are two work positions, at which four 3-dof NC positioners support a carrier which fix a fuselage. Each mechanism at a work position is a typically distributed and overconstrained 4-PPPS parallel mechanism. Compared with the serial type mechanism, the overconstrained parallel mechanism can bear heavy load and achieve higher stiffness, for there are four formal chains to apply supporting forces to the carrier. However, the character also results in low accuracy, for there are many joints participating in the system motion especially for the docking system which is large, separately assembled with relatively high manufacturing errors [1]. Taking the NC positioner for example, the installation error between the designed positions and the real positions is inevitably. And, the moving axes of them are very hard to be installed absolutely parallel to each other.

To improve the accuracy of the docking system, calibration is required to identify the real structural parameters [2]. For some kind of parallel mechanism [3-6], the joints would not work together very well because of the manufacturing and assembly errors. The joints should overcome the internal forces which force the mechanism to form minor deformation. Under this case, the motion error includes two main factors including mechanism error and deformation error. So, a kind of calibration method [3] was proposed to estimate the deformation error of the end effector when approximating the mechanism parameters. An additional virtual revolute joint was added to the mechanism to simulate the deformation. And, Jiang et al. [4] added additional deformation compatibility equations into the force balance equation to derive the deformation of the end effector. Ecorchard et al. [5] disassembled deformations of 
the end effector of the overconstrained mechanism as the sum of the poses derived from the geometry-based kinematic models of equivalent non-overconstrained mechanisms.

To calibrate the structure parameters, a kinematic model or structure character should be established firstly. Motion chain [6-8] is a mainstream method to describe the motion vector relationship of a branch chain in the parallel mechanism. This method has a clear and specific space geometry which is easy to understand but with ambiguous kinematic description. The singular position problem would also affect the calibration accuracy, for the ill matrix leads to wrong inverse kinematic result. Liu estimation [9] was used in the calibration process to resolve the multicollinearity in the identification Jacobian. To simplify the calculation difficulty and to decrease the value range of the calibrated parameters, the product of exponential (POE) formula was used to describe the closed-chain structure of the parallel mechanism [10]. But, some imperfect geometric errors, such as the axes offset in the universal joints [11], cannot be taken into account in the system calibration models. And, in fact, POE is another mathematical form of the motion chain method.

After system structure modelling with motion errors, it is very important to calculate the optimal parameters. The parameters of the calibration equations were usually estimated by the linear least square method [12]. Neural network [13,14] and gene algorithm [15] are creatively used in the solution procedure. But, the training process of neural network needs a large amount of data which necessitates the researcher to measure thousands of joint positions and corresponding end effector poses and postures. As the number of the parameters to be solved increases, the efficiency of the gene algorithm decreases dramatically.

In terms of improving the accuracy of the docking system, Hamid and Heikki [16] used the Monte Carlo method to estimate the optimal docking pose and posture which were fed back by an optical measurement system. But, for a mechanism with a structure error, not only the realtime closed-loop control method does any help to promote the accuracy but also it would increase the risk of system shock [17]. The influence of aircraft coordinate on the docking error was analyzed by Jin et al. [18]. His research focus on different arrangement forms of datum points leads to different matching accuracies. But, he assumed that different forms of datum points are located at the principle points which are nearly impossible in practical engineering. How to calculate the aircraft coordinate with some inaccurate datum points is a problem to be solved.

\section{System Structure and Ground Datum Calibration}

2.1. System Structure. The designed aircraft docking system is used in the assemble process of the aft and rear fuselages. The main structure of the docking system is shown in Figure 2. It includes three main functional modules, such as ground datum module, NC positioners, and carriers.

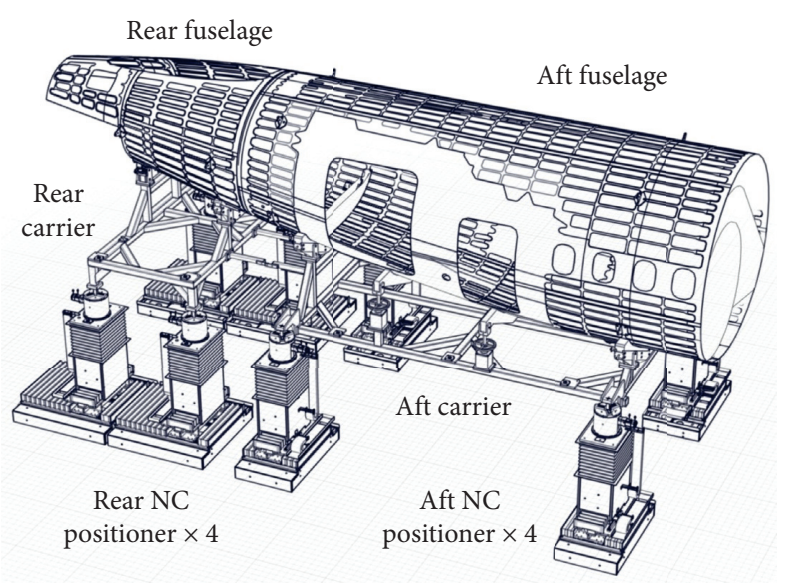

FIgUre 1: Airplane fuselage docking system.

The ground datum module is composed of 14 standard datum points which are designed to match the plane coordinate. When the air plane is designed, there is a hypothetical uniform coordinate called plane coordinate which is the reference in the entire assemble and inspection process. Every time the two fuselages of a plane are assembled, the object of the work is to move the two fuselages to the predefined position and posture in the plane coordinate, respectively, and joint them together.

The two fuselages correspond to two work positions. At each work position, a carrier that fixes the fuselage is supported by four NC positioners that form a linkage system. Each positioner has three prismatic pairs. When the four positioners move coordinated, the fuselage changes its position and posture with its supporting carrier. Spherical linkages are used to connect the positioners and the carrier at the supporting points. It is a typical 4-PPPS parallel structure.

2.2. Ground Datum Calibration. The original point of the plane coordinate is defined along the intersection line of the engine room floor plane and the symmetry plane of the aircraft and $550 \mathrm{~mm}$ before the airplane nose. Actually, the airplane coordinate cannot be found directly in the docking system. But as required, the docking position and pilot process should be recorded in the plane system. So, a set of reference points called the ground datum points are defined in the airplane coordinate fixed on the ground to confirm the relationship between the airplane and measurement coordinates.

The principle positions of the ground datum points are designed together with the docking system. But, in the construction process of the mechanism, the real ground datum points cannot be localized very accurately. Usually, the principle and real positions have large deviations which can reach several centimeters. So it is very important to measure and calculate the real position values of the ground datum points. If the position values of the ground datum are accurate, the matching matrix between the airplane coordinate and measurement coordinate can be accurate. 


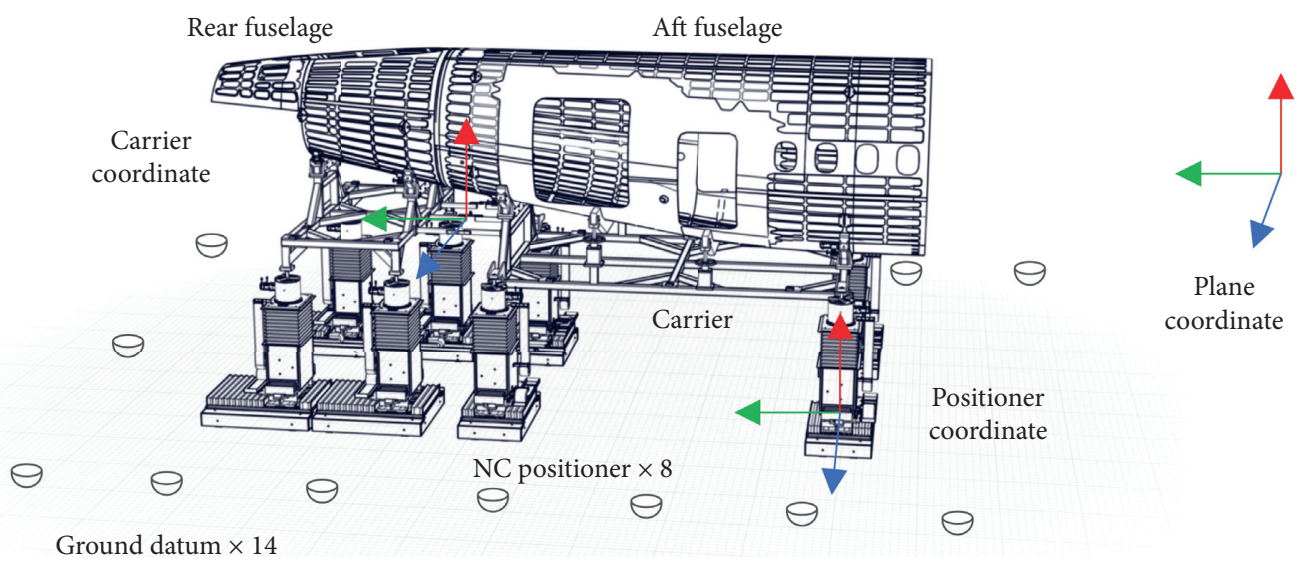

Figure 2: Main structure of the docking system.

The airplane coordinate is defined as $C_{w}$, and the principle point-group of the ground datum is $Q_{i}(i=1,2,3$, ...). Before the calibration process, it is necessary to measure the ground datum points several times from different directions. Even though the laser tracker is very accurate as known to all, large range measurement can cause accuracy degradation with $0.01 \mathrm{~mm} / \mathrm{m}$. Multiple metering can help to reduce the error effect. It is assumed that the measurement coordinate of the laser tracker is $C_{m, j}$, in which $j$ denotes the $j$ th measurement. And, the point-groups $P_{i, j}(i=1,2,3, \ldots)$ denote the real measurement points in the $j$ th measurement coordinate. We consider the first measurement coordinate $C_{m, 1}$ as the reference coordinate.

$P_{i, j}(i=1,2,3, \ldots, j=2,3, \ldots)$ and $P_{i, 1}(i=1,2,3, \ldots)$ are two similar point-groups defined in different measurement coordinates. As the two point-groups are similar, they can be matched. And, the augmented transform matrix $M_{p j}^{p 1}$ from $P_{i, j}$ to $P_{i, 1}$ can be written as

$$
M_{p j}^{p 1}=\left[\begin{array}{rrrr} 
& R & T \\
0 & 0 & 0 & 1
\end{array}\right],
$$

where $R$ is the rotation matrix and $T$ is the translation vector. The matching of the two point-groups is to minimize the cost function which can be written as

$$
\min E=\sum_{i=1}^{n}\left\|P_{i, 1}-\left(P_{i, j} \cdot R+T\right)\right\|^{2} .
$$

Then, a matching problem is transformed to an optimization problem which can be solved by Newton iteration [19]. Then, with the transform matrix $M_{p j}^{p 1}$, the measurement pointgroup $P_{i, j}$ in $C_{m, j}$ can be mapped to the reference measurement coordinate $C_{m, 1}$, and new point-group $P_{i, 1}^{j}$ is obtained:

$$
P_{i, 1}^{j}=M_{p j}^{p 1} \cdot P_{i, j}, \quad i=1,2,3, \ldots, j=1,2,3, \ldots,
$$

where

$$
\begin{aligned}
& P_{i, 1}^{j}=\left[x_{i, 1}^{j}, y_{i, 1}^{j}, z_{i, 1}^{j}\right]^{\prime}, \\
& P_{i, 1}^{1}=P_{i, 1} .
\end{aligned}
$$

For the existence of the measurement error, pointgroups $P_{i, 1}^{j}(j=1,2,3, \ldots)$ are similar but not exactly the same. So, the expectation of every point in the point-groups can be calculated by the mean value. The optical estimation of the ground datum points $\bar{P}_{i}$ can be written as

$$
\bar{P}_{i}=\left[\begin{array}{c}
\bar{x}_{i} \\
\bar{y}_{i} \\
\bar{z}_{i}
\end{array}\right]=\left[\begin{array}{l}
\frac{\sum_{j=1}^{n} x_{i, 1}^{j}}{n} \\
\frac{\sum_{j=1}^{n} y_{i, 1}^{j}}{n} \\
\frac{\sum_{j=1}^{n} z_{i, 1}^{j}}{n}
\end{array}\right] .
$$

where $n$ is the total number of measurements. With the optical estimation $\bar{P}_{i}$ of the datum points measured in the reference measurement coordinate, the principle coordinate value of the ground datum points $Q_{i}$ can be revised. The flowchart of the calibration steps is shown in Figure 3, where $\Delta$ is the maximal value of the matching error.

\section{Kinematics and Mechanism Calibration Method}

3.1. Kinematics and Error Analysis. We take the rear carrier as an example; the carrier is a rigid, lightly tortured frame structure, for the machining process cannot guarantee its accuracy. It is assumed that the real-time spatial positions of the four spherical linkages in the airplane coordinate are $P_{i}^{w}$ $(i=1,2,3,4)$

The inverse kinematics is to calculate the actual displacements of every positioner by the known carrier position and posture. It is assumed that, at one time, the position and posture matrix of the carrier in the airplane coordinate is $M_{c}$ :

$$
T_{c}^{w}=\left[\begin{array}{cc}
R_{c}^{w} & P_{c} \\
0_{1 \times 3} & 1
\end{array}\right] .
$$

As the carrier is seen as a rigid body, the relationships between the four spherical linkages and the original point of the carrier coordinate should be constant vectors which can be written as $v_{p i}^{c}(i=1,2,3,4)$ : 


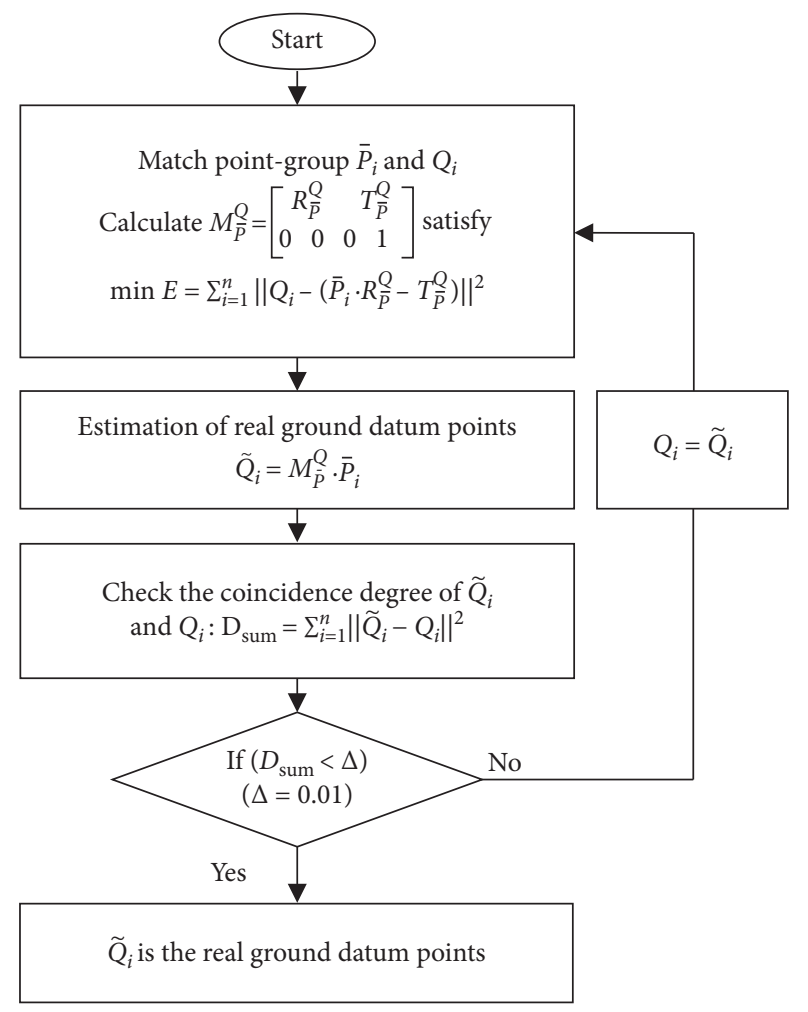

FIGURE 3: Ground datum point calibration algorithm.

$$
\left[\begin{array}{c}
P_{i}^{w} \\
1
\end{array}\right]=T_{c}^{w} \cdot\left[\begin{array}{c}
v_{p i}^{c} \\
1
\end{array}\right], \quad i=1,2,3,4
$$

where $P_{i}^{w}$ is the position vector of the $i$-th positioner in the plane coordinate. The displacements of each axis in the positioner coordinate is

$$
P_{i}^{p}=\left(T_{P i}^{w}\right)^{-1} \cdot P_{i}^{w}
$$

where $T_{P i}^{w}$ is the transformation matrix from the coordinate of the $i$-th positioner to the airplane coordinate.

In equation (7), $T_{c}^{w}$ can be obtained by a measurement process before docking. However, $v_{p i}^{c}$ which has three parameters $\left(x_{p i}^{c}, y_{p i}^{c}, z_{p i}^{c}\right)$ should have some error between its principle value and its real value for the machining error of the carrier. The vector $P_{i}^{w}$ is not that accurate for it is got by the multiplication of an accurate measured matrix $T_{c}^{w}$ and an inaccurate vector $v_{p i}^{c}$. In equation (12), the principle value of $T_{P i}^{w}$ should be not very accurate for the existence of the installation error of the positioners. There are 12 parameters to be determined in the matrix $T_{P i}^{w}$ (Figure 4).

3.2. Kinematics Calibration. A calibration coordinate is established on a tooling that fixes the target ball of the laser tracker. The transform matrix from the calibration coordinate $\left\{C_{c}\right\}$ to the airplane coordinate $\left\{C_{w}\right\}$ is $T_{c}^{w}$ which can be determined by the design model. The origin of the $i$ th positioner coordinate $\left\{C_{p i}\right\}$ is located at the center of the spherical linkage as shown in Figure 5. The transform matrix from the positioner coordinate $\left\{C_{p i}\right\}$ to the airplane coordinate $\left\{C_{w}\right\}$ is $T_{p i}^{w}$. It is necessary to state that even though in the designed model the origins of the four positioner coordinates are the four vertices of a plane rectangle and the corresponding axes are parallel to each other. However, in the real condition, the carrier is a large low-accuracy weldment where spatial warping exists and the installation of the mechanism cannot guarantee the design accuracy as well. As a matter of fact, the axes of the four positioner coordinates are not parallel to each other. Their coordinate frame represented in airplane coordinate should be calibrated.

When the $i$ th positioner moves, there are two methods to denote its position in the airplane coordinate $P_{i}^{w}$. One is by the positioner coordinate [20]:

$$
\left[p_{i}^{w}, 1\right]^{T}=T_{p i}^{w} \cdot\left[v^{p i}, 1\right]^{T}
$$

where $v^{p i}=\left[l_{i x}, l_{i y}, l_{i z},\right]$ is the axis movement vector in the positioner coordinate. And, the other method is by the calibration coordinate:

$$
\left[p_{i}^{w}, 1\right]^{T}=T_{c}^{w} \cdot\left[v_{p i}^{c}, 1\right]^{T},
$$

where $v_{p i}^{c}$ is the vector from the original point of the calibration coordinate to the sphere link of the $i$ th positioner coordinate. For the carrier is assumed to be a rigid body, the vector $v_{p i}^{c}$ is a constant value no matter how the mechanism moves.

By equations (9) and (10), the following equation can be obtained:

$$
T_{p i}^{w} \cdot\left[v^{p i}, 1\right]^{T}=T_{c}^{w} \cdot\left[v_{p i}^{c}, 1\right]^{T}
$$

Extending equation (11), we get

$$
\left[\begin{array}{cccc}
e_{i x}^{w} & e_{i y}^{w} & e_{i z}^{w} & d_{i}^{w} \\
0 & 0 & 0 & 1
\end{array}\right] \cdot\left[\begin{array}{c}
l_{i x} \\
l_{i y} \\
l_{i z} \\
1
\end{array}\right]=\left[\begin{array}{cc}
R_{c}^{w} & P_{c} \\
0_{1 \times 3} & 1
\end{array}\right] \cdot\left[\begin{array}{c}
v_{p i}^{c} \\
1
\end{array}\right],
$$

where $e_{i x}^{w}, e_{i y}^{w}$, and $e_{i z}^{w}$ are the three unit vectors in $T_{p i}^{w}$, which denote the axes directions of the ith positioner in airplane coordinate, $d_{i}^{w}$ is the original position of the $i$ th positioner in airplane coordinate, And $R_{c}^{w}$ and $P_{c}$ are the rotation matrix and translation vector of $T_{c}^{w}$. In the calibration process, $R_{c}^{w}$ and $P_{c}$ change with the motion of the mechanism. Their value can be obtained by the feedbacks of the laser tracker; $v_{p i}^{c}$ is a constant vector with error.

Deriving equation (12), we get

$$
\begin{aligned}
& {\left[\begin{array}{cccc}
e_{i x}^{w} & e_{i y}^{w} & e_{i z}^{w} & d_{i}^{w} \\
0 & 0 & 0 & 1
\end{array}\right]^{\prime} \cdot\left[\begin{array}{c}
l_{i x} \\
l_{i y} \\
l_{i z} \\
1
\end{array}\right]+\left[\begin{array}{cccc}
e_{i x}^{w} & e_{i y}^{w} & e_{i z}^{w} & d_{i}^{w} \\
0 & 0 & 0 & 1
\end{array}\right] \cdot\left[\begin{array}{c}
l_{i x} \\
l_{i y} \\
l_{i z} \\
1
\end{array}\right]^{\prime}} \\
& =\left[\begin{array}{cc}
R_{c}^{w} & P_{c} \\
0_{1 \times 3} & 1
\end{array}\right]^{\prime} \cdot\left[\begin{array}{c}
v_{p i}^{c} \\
1
\end{array}\right]+\left[\begin{array}{cc}
R_{c}^{w} & P_{c} \\
0_{1 \times 3} & 1
\end{array}\right] \cdot\left[\begin{array}{c}
v_{p i}^{c} \\
1
\end{array}\right]^{\prime} \text {. }
\end{aligned}
$$

For the left-hand side of equation (13), the two items can be written as 


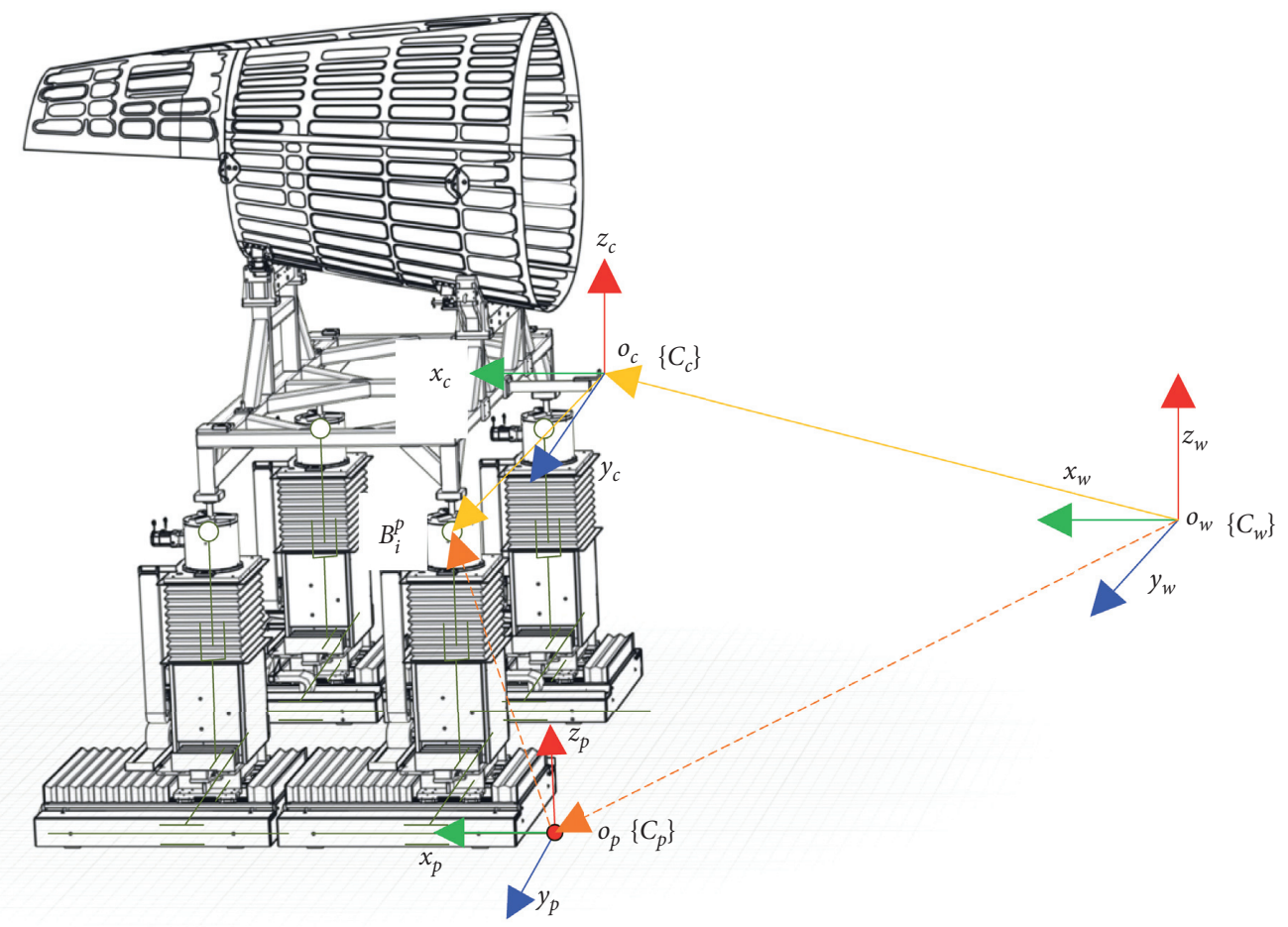

FIgURE 4: Coordinate relationships of the docking system.

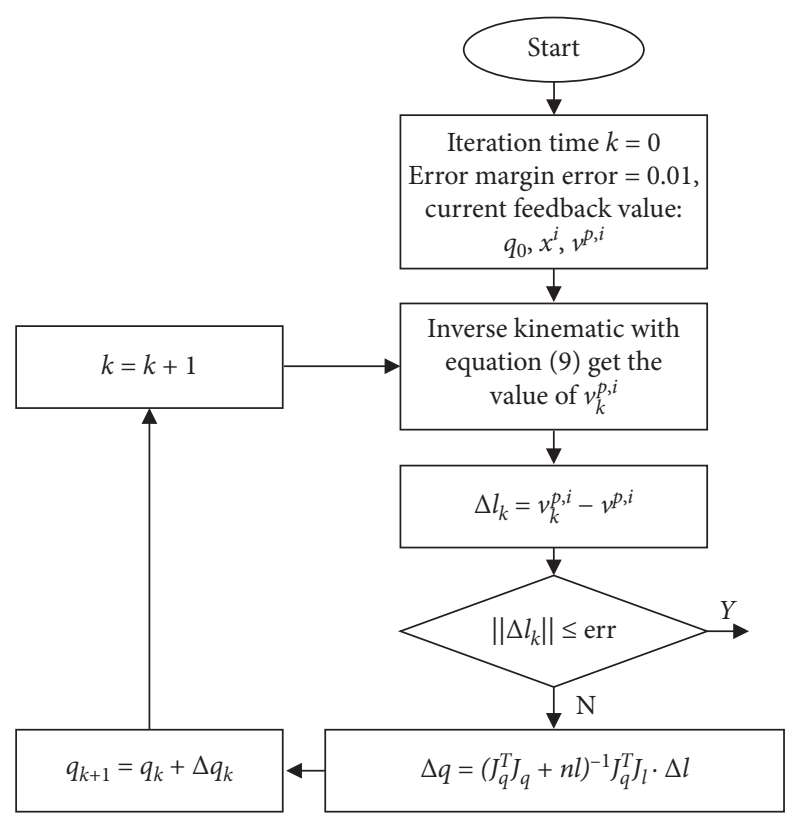

FIGURE 5: Flow chart of the calibration process.

$$
\begin{aligned}
& {\left[\begin{array}{cccc}
e_{i x}^{w} & e_{i y}^{w} & e_{i z}^{w} & d_{i}^{w} \\
0 & 0 & 0 & 1
\end{array}\right]^{\prime} \cdot\left[\begin{array}{c}
l_{i x} \\
l_{i y} \\
l_{i z} \\
1
\end{array}\right]=\delta e_{i x}^{w} \cdot l_{i x}+\delta e_{i y}^{w} \cdot l_{i y}+\delta e_{i z}^{w} \cdot l_{i z}+\delta d_{i}^{w},} \\
& {\left[\begin{array}{cccc}
e_{i x}^{w} & e_{i y}^{w} & e_{i z}^{w} & d_{i}^{w} \\
0 & 0 & 0 & 1
\end{array}\right] \cdot\left[\begin{array}{c}
l_{i x} \\
l_{i y} \\
l_{i z} \\
1
\end{array}\right]=\left[\begin{array}{cccc}
e_{i x}^{w} & e_{i y}^{w} & e_{i z}^{w} & d_{i}^{w} \\
0 & 0 & 0 & 1
\end{array}\right] \cdot\left[\begin{array}{c}
\delta l_{i x} \\
\delta l_{i y} \\
\delta l_{i z} \\
0
\end{array}\right]=e_{i x}^{w} \cdot \delta l_{i x}+e_{i y}^{w} \cdot \delta l_{i y}+e_{i z}^{w} \cdot \delta l_{i z} \cdot}
\end{aligned}
$$


On the right-hand side of equation (13),

$$
\left[\begin{array}{cc}
R_{c}^{w} & P_{c} \\
0_{1 \times 3} & 1
\end{array}\right]^{\prime}=\Delta_{c}^{w} \cdot\left[\begin{array}{cc}
R_{c}^{w} & P_{c} \\
0_{1 \times 3} & 1
\end{array}\right]
$$

where

$$
\Delta_{c}^{w}=\left[\begin{array}{cccc}
0 & -\delta \theta_{z} & \delta \theta_{y} & \delta p_{x} \\
\delta \theta_{z} & 0 & -\delta \theta_{x} & \delta p_{y} \\
-\delta \theta_{y} & \delta \theta_{x} & 0 & \delta p_{z} \\
0 & 0 & 0 & 0
\end{array}\right]=\left[\begin{array}{cc}
\delta \hat{\theta} & \delta p \\
0_{1 \times 3} & 0
\end{array}\right] .
$$

So, the first item for the right-hand side of equation (13) can be written as

$$
\begin{aligned}
{\left[\begin{array}{cc}
R_{c}^{w} & P_{c} \\
0_{1 \times 3} & 1
\end{array}\right]^{\prime} \cdot\left[\begin{array}{c}
v_{p i}^{c} \\
1
\end{array}\right] } & =\Delta_{c}^{w} \cdot\left[\begin{array}{cc}
R_{c}^{w} & P_{c} \\
0_{1 \times 3} & 1
\end{array}\right] \cdot\left[\begin{array}{c}
v_{p i}^{c} \\
1
\end{array}\right] \\
& =\left[\begin{array}{cc}
\delta \hat{\theta} \cdot R_{c}^{w} & \delta \hat{\theta} \cdot \delta p \\
0_{1 \times 3} & \delta p
\end{array}\right] \cdot\left[\begin{array}{c}
v_{p i}^{c} \\
1
\end{array}\right] .
\end{aligned}
$$

For the diagonal elements of the matrix $\delta \hat{\theta}$ are all zero, the item

$$
\delta \widehat{\theta} \cdot \delta p=0_{3 \times 1}
$$

So,

$$
\left[\begin{array}{cc}
R_{c}^{w} & P_{c} \\
0_{1 \times 3} & 1
\end{array}\right]^{\prime} \cdot\left[\begin{array}{c}
v_{p i}^{c} \\
1
\end{array}\right]=\delta \widehat{\theta} \cdot R_{c}^{w} \cdot v_{p i}^{c}+\delta p .
$$

The second item for the right-hand side of equation (13) can be written as

$$
\left[\begin{array}{cc}
R_{c}^{w} & P_{c} \\
0_{1 \times 3} & 1
\end{array}\right] \cdot\left[\begin{array}{c}
v_{p i}^{c} \\
1
\end{array}\right]=\left[\begin{array}{cc}
R_{c}^{w} & P_{c} \\
0_{1 \times 3} & 1
\end{array}\right] \cdot\left[\begin{array}{c}
\delta v_{p i}^{c} \\
0
\end{array}\right]=R_{c}^{w} \cdot \delta v_{p i}^{c} .
$$

According to equations (14), (15), (20), and (21), equation (13) can be written as

$$
\begin{aligned}
\delta e_{i x}^{w} & l_{i x}+\delta e_{i y}^{w} \cdot l_{i y}+\delta e_{i z}^{w} \cdot l_{i z}+e_{i x}^{w} \cdot \delta l_{i x} \\
& +e_{i y}^{w} \cdot \delta l_{i y}+e_{i z}^{w} \cdot \delta l_{i z}+\delta d_{i}^{w} \\
= & \delta \hat{\theta} \cdot R_{c}^{w} \cdot v_{p i}^{c}+\delta p+R_{c}^{w} \cdot \delta v_{p i}^{c} .
\end{aligned}
$$

On both sides of equation (22), multiply $\left(e_{i x}^{w}\right)^{T},\left(e_{i y}^{w}\right)^{T}$, and $\left(e_{i z}^{w}\right)^{T}$, respectively. For the three vectors $e_{i x}^{w}, e_{i \mathrm{y}}^{w}$, and $e_{i z}^{w}$ are spatial unit vectors, $\left(e_{i x}^{w}\right)^{T} \cdot e_{i x}^{w}=1, \quad\left(e_{i y}^{w}\right)^{T} \cdot e_{i y}^{w}=1$, $\left(e_{i z}^{w}\right)^{T} \cdot e_{i z}^{w}=1$ and $\left(e_{i x}^{w}\right)^{T} \cdot \delta e_{i x}^{w}=1, \quad\left(e_{i y}^{w}\right)^{T} \cdot \delta e_{i y}^{w}=1, \quad\left(e_{i z}^{w}\right)^{T}$. $\delta e_{i z}^{w}=1$. So, an equation set for the $i$ th positioner can be written as

$$
\left\{\begin{array}{l}
\left(e_{i x}^{w}\right)^{T} \delta e_{i y}^{w} \cdot l_{i y}+\left(e_{i x}^{w}\right)^{T} \delta e_{i z}^{w} \cdot l_{i z}+\delta l_{i x}+\left(e_{i x}^{w}\right)^{T} \delta e_{i y}^{w} \cdot \delta l_{i y}+\left(e_{i x}^{w}\right)^{T} e_{i z}^{w} \cdot \delta l_{i z} \\
=\left(v_{p i}^{c} \times e_{i x}^{w}\right)^{T} \delta \widehat{\theta}+\left(e_{i x}^{w}\right)^{T} \delta p+\left(e_{i x}^{w}\right)^{T} R_{c}^{w} \cdot \delta v_{p i}^{c}-\left(e_{i x}^{w}\right)^{T} \delta d_{i}^{w}\left(e_{i y}^{w}\right)^{T} \delta e_{i x}^{w} \cdot l_{i x}+\left(e_{i y}^{w}\right)^{T} \delta e_{i z}^{w} \cdot l_{i z}+\left(e_{i y}^{w}\right)^{T} e_{i x}^{w} \cdot \delta l_{i x}+\delta l_{i y}+\left(e_{i y}^{w}\right)^{T} e_{i z}^{w} \cdot \delta l_{i z} \\
=\left(v_{p i}^{c} \times e_{i y}^{w}\right)^{T} \delta \hat{\theta}+\left(e_{i y}^{w}\right)^{T} \delta p+\left(e_{i y}^{w}\right)^{T} R_{c}^{w} \cdot \delta v_{p i}^{c}-\left(e_{i y}^{w}\right)^{T} \delta d_{i}^{w}\left(e_{i z}^{w}\right)^{T} \delta e_{i x}^{w} \cdot l_{i x}+\left(e_{i z}^{w}\right)^{T} \delta e_{i y}^{w} \cdot l_{i y}+\left(e_{i z}^{w}\right)^{T} e_{i x}^{w} \cdot \delta i_{i x}+\left(e_{i z}^{w}\right)^{T} \delta e_{i y}^{w} \cdot \delta l_{i y}+\delta l_{i z} \\
=\left(v_{p i}^{c} \times e_{i z}^{w}\right)^{T} \delta \hat{\theta}+\left(e_{i z}^{w}\right)^{T} \delta p+\left(e_{i y}^{w}\right)^{T} R_{c}^{w} \cdot \delta v_{p i}^{c}-\left(e_{i z}^{w}\right)^{T} \delta d_{i}^{w} .
\end{array}\right.
$$

Equation (23) can be written in a matrix form:

$$
\begin{gathered}
{\left[\begin{array}{ccc}
1 & \left(e_{i x}^{w}\right)^{T} e_{i y}^{w} & \left(e_{i x}^{w}\right)^{T} e_{i z}^{w} \\
\left(e_{i y}^{w}\right)^{T} e_{i x}^{w} & 1 & \left(e_{i y}^{w}\right)^{T} e_{i z}^{w} \\
\left(e_{i z}^{w}\right)^{T} e_{i x}^{w} & \left(e_{i z}^{w}\right)^{T} e_{i y}^{w} & 1
\end{array}\right]\left[\begin{array}{l}
\delta l_{i x} \\
\delta l_{i y} \\
\delta l_{i z}
\end{array}\right]+\left[\begin{array}{ccc}
0 & \left(e_{i x}^{w}\right)^{T} l_{i y} & \left(e_{i x}^{w}\right)^{T} l_{i z} \\
\left(e_{i y}^{w}\right)^{T} l_{i x} & 0 & \left(e_{i y}^{w}\right)^{T} l_{i z} \\
\left(e_{i z}^{w}\right)^{T} l_{i x} & \left(e_{i z}^{w}\right)^{T} l_{i y} & 0
\end{array}\right]\left[\begin{array}{l}
\delta e_{i x}^{w} \\
\delta e_{i y}^{w} \\
\delta e_{i z}^{w}
\end{array}\right]} \\
=\left[\begin{array}{ll}
\left(v_{p i}^{c} \times e_{i x}^{w}\right)^{T} & \left(e_{i x}^{w}\right)^{T} \\
\left(v_{p i}^{c} \times e_{i y}^{w}\right)^{T} & \left(e_{i y}^{w}\right)^{T} \\
\left(v_{p i}^{c} \times e_{i z}^{w}\right)^{T} & \left(e_{i z}^{w}\right)^{T}
\end{array}\right]\left[\begin{array}{l}
\delta \hat{\theta} \\
\delta p
\end{array}\right]+\left[\begin{array}{cc}
-\left(e_{i x}^{w}\right)^{T} R_{c}^{w} & \left(e_{i x}^{w}\right)^{T} \\
-\left(e_{i y}^{w}\right)^{T} R_{c}^{w} & \left(e_{i y}^{w}\right)^{T} \\
-\left(e_{i z}^{w}\right)^{T} R_{c}^{w} & \left(e_{i z}^{w}\right)^{T}
\end{array}\right]\left[\begin{array}{l}
\delta v_{p i}^{c} \\
\delta d_{i}^{w}
\end{array}\right] .
\end{gathered}
$$

where $\left[\begin{array}{lll}\delta l_{i x} & \delta l_{i y} & \delta l_{i z}\end{array}\right]^{T}$ are the incremental distances of the three axes on the ith positioner and $\left[\begin{array}{ll}\delta \widehat{\theta} & \delta \mathrm{p}\end{array}\right]^{T}$ are the angle and position variations of the carrier coordinate to be measured in the calibration process. These two vectors can be measured accurately by the feedback sensors and the laser tracker in the docking system. $\left[\begin{array}{lll}\delta e_{i \mathrm{x}}^{w} & \delta e_{i \mathrm{y}}^{w} & \delta e_{i z}^{w}\end{array}\right]^{T}$ and $\left[\begin{array}{ll}\delta v_{p i}^{c} & \delta d_{i}^{w}\end{array}\right]^{T}$ are two unknown vectors to be calibrated. $\delta e_{i \mathrm{x}}^{w}$, 
$\delta e_{i \mathrm{y}}^{w}$, and $\delta e_{i z}^{w}$ denote the direction deviation of the three axes on the $i$ th positioner. $\delta d_{i}^{w}$ is the position error of the original point for the $i$ th positioner. $\delta v_{p i}^{c}$ is the error of the constraint vector which denotes the relationship between the $i$ th positioner and the carrier coordinate to be measured in the calibration process.

Equation (24) denotes the kinematic differential relation between just one positioner and the carrier coordinate measured in the calibration process. It can be written in a simplified mode:

$$
J_{l, i} \cdot \delta l_{i}+J_{e, i} \cdot \delta e_{i}-J_{h, i} \cdot \delta h_{i}=J_{p, i} \cdot \delta x .
$$

where

$$
\begin{aligned}
& J_{l, i}=\left[\begin{array}{ccc}
1 & \left(e_{i x}^{w}\right)^{T} e_{i y}^{w} & \left(e_{i x}^{w}\right)^{T} e_{i z}^{w} \\
\left(e_{i y}^{w}\right)^{T} e_{i x}^{w} & 1 & \left(e_{i y}^{w}\right)^{T} e_{i z}^{w} \\
\left(e_{i z}^{w}\right)^{T} e_{i x}^{w} & \left(e_{i z}^{w}\right)^{T} e_{i y}^{w} & 1
\end{array}\right], \\
& \delta l_{i}=\left[\begin{array}{l}
\delta l_{i x} \\
\delta l_{i y} \\
\delta l_{i z}
\end{array}\right] \\
& J_{e, i}=\left[\begin{array}{ccc}
0 & \left(e_{i x}^{w}\right)^{T} l_{i y} & \left(e_{i x}^{w}\right)^{T} l_{i z} \\
\left(e_{i y}^{w}\right)^{T} l_{i x} & 0 & \left(e_{i y}^{w}\right)^{T} l_{i z} \\
\left(e_{i z}^{w}\right)^{T} l_{i x} & \left(e_{i z}^{w}\right)^{T} l_{i y} & 0
\end{array}\right], \\
& \delta e_{i}=\left[\begin{array}{c}
\delta e_{i x}^{w} \\
\delta e_{i y}^{w} \\
\delta e_{i z}^{w}
\end{array}\right], \\
& J_{h, i}=\left[\begin{array}{cc}
-\left(e_{i x}^{w}\right)^{T} R_{c}^{w} & \left(e_{i x}^{w}\right)^{T} \\
-\left(e_{i y}^{w}\right)^{T} R_{c}^{w} & \left(e_{i y}^{w}\right)^{T} \\
-\left(e_{i z}^{w}\right)^{T} R_{c}^{w} & \left(e_{i z}^{w}\right)^{T}
\end{array}\right], \\
& \delta h_{i}=\left[\begin{array}{l}
\delta v_{p i}^{c} \\
\delta d_{i}^{w}
\end{array}\right], \\
& J_{p, i}=\left[\begin{array}{ll}
\left(v_{p i}^{c} \times e_{i x}^{w}\right)^{T} & \left(e_{i x}^{w}\right)^{T} \\
\left(v_{p i}^{c} \times e_{i y}^{w}\right)^{T} & \left(e_{i y}^{w}\right)^{T} \\
\left(v_{p i}^{c} \times e_{i z}^{w}\right)^{T} & \left(e_{i z}^{w}\right)^{T}
\end{array}\right], \\
& \delta x=\left[\begin{array}{l}
\delta \hat{\theta} \\
\delta p
\end{array}\right] \text {. }
\end{aligned}
$$

To calibrate the four positioners at a time, equation (24) should be expanded to a completely matrix:

$$
J_{l} \cdot \delta l+J_{e} \cdot \delta e-J_{h} \cdot \delta h=J_{p} \cdot \delta x .
$$

where

$$
J_{l}=\left[\begin{array}{cccc}
J_{l, 1} & 0 & 0 & 0 \\
0 & J_{l, 2} & 0 & 0 \\
0 & 0 & J_{l, 3} & 0 \\
0 & 0 & 0 & J_{l, 4}
\end{array}\right],
$$$$
\delta l=\left[\begin{array}{l}
\delta l_{1} \\
\delta l_{2} \\
\delta l_{3} \\
\delta l_{4}
\end{array}\right] \text {, }
$$$$
J_{e}=\left[\begin{array}{cccc}
J_{e, 1} & 0 & 0 & 0 \\
0 & J_{e, 2} & 0 & 0 \\
0 & 0 & J_{e, 3} & 0 \\
0 & 0 & 0 & J_{e, 4}
\end{array}\right] \text {, }
$$$$
\delta e=\left[\begin{array}{l}
\delta e_{1} \\
\delta e_{2} \\
\delta e_{3} \\
\delta e_{4}
\end{array}\right]
$$$$
J_{h}=\left[\begin{array}{cccc}
J_{h, 1} & 0 & 0 & 0 \\
0 & J_{h, 2} & 0 & 0 \\
0 & 0 & J_{h, 3} & 0 \\
0 & 0 & 0 & J_{h, 4}
\end{array}\right] \text {, }
$$$$
\delta h=\left[\begin{array}{l}
\delta h_{1} \\
\delta h_{2} \\
\delta h_{3} \\
\delta h_{4}
\end{array}\right],
$$$$
J_{p}=\left[\begin{array}{c}
J_{p, 1} \\
J_{p, 2} \\
J_{p, 3} \\
J_{p, 4}
\end{array}\right] \text {, }
$$$$
\delta x=\left[\begin{array}{l}
\delta \widehat{\theta} \\
\delta p
\end{array}\right]
$$

3.3. Calibration Iteration. For a calibration calculation process, equation (27) should be written as $\delta x=J_{p}^{-1}\left[J_{l} \cdot \delta l-\left(J_{h} \cdot \delta h-J_{e} \cdot \delta e\right)\right]$, which can be further simplified into

$$
\delta x=J_{p}^{-1}\left[J_{l} \cdot \delta l-J_{q} \cdot \delta q\right] .
$$

where $J_{q}=\left[\begin{array}{ll}J_{h} & -J_{e}\end{array}\right]$ and

$$
\delta q=\left[\begin{array}{ll}
\delta h & \delta e
\end{array}\right]^{T} .
$$

Even though the measurement error $\delta x$ is inevitable, the accuracy of the laser tracker is trustworthy. So, the ideal least square solution of equation (28) is 


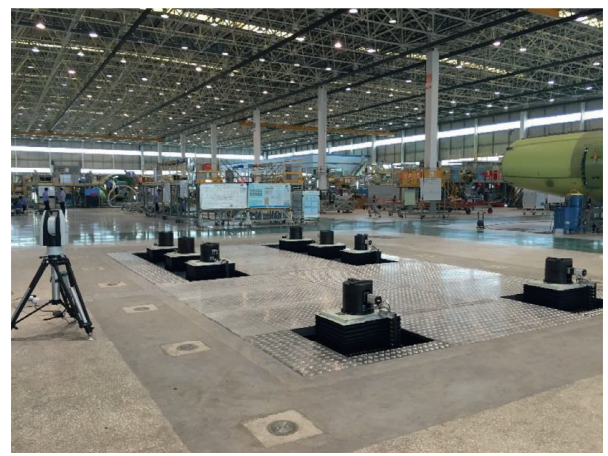

Figure 6: Ground datum point measurement.

TABLE 1: Ground datum points (unit: $\mathrm{mm}$ ).

\begin{tabular}{lccccc}
\hline No & Principle value & Practical value 1 & Practical value 2 & Practical value 3 \\
\hline 1 & {$[193003500-2220]$} & {$[-3156.260-8155.329-1392.723]$} & {$[-3058.413-8053.097-1402.116]$} & {$[-1159.827-8512.139-1407.106]$} \\
2 & {$[213003500-2220]$} & {$[-1156.309-8285.712-1401.025]$} & {$[-1056.119-8140.399-1410.115]$} & {$[809.969-8142.388-1413.311]$} \\
3 & {$[233003500-2220]$} & {$[2826.598-8613.591-1411.899]$} & {$[2932.833-8382.425-1422.264]$} & {$[4749.681-7472.045-1422.035]$} \\
4 & {$[253003500-2220]$} & {$[4823.645-8737.690-1417.086]$} & {$[4932.103-8463.510-1428.166]$} & {$[6715.110-7096.895-1425.733]$} \\
5 & {$[273003500-2220]$} & {$[6832.038-8878.672-1419.055]$} & {$[6942.950-8561.236-1431.004]$} & {$[8695.685-6735.304-1426.707]$} \\
6 & {$[293003500-2220]$} & {$[8833.812-9021.330-1425.487]$} & {$[8947.387-8660.739-1438.082]$} & {$[10670.312-6376.855-1431.975]$} \\
7 & {$[313003500-2220]$} & {$[9084.539-5533.065-1423.092]$} & {$[9122.903-5167.949-1432.217]$} & {$[10047.936-2935.490-1424.554]$} \\
8 & {$[313000-2220]$} & {$[9331.277-2022.783-1432.762]$} & {$[9294.040-1653.241-1437.728]$} & {$[9416.222526 .270-1429.161]$} \\
9 & {$[31300-3500-2220]$} & {$[7310.028-1917.118-1431.633]$} & {$[7271.011-1591.021-1435.883]$} & {$[7431.948127 .310-1429.307]$} \\
10 & {$[29300-3500-2220]$} & {$[5301.887-1753.677-1431.180]$} & {$[5259.813-1470.810-1434.658]$} & {$[5446.019-212.500-1430.045]$} \\
11 & {$[27300-3500-2220]$} & {$[3326.476-1621.557-1426.317]$} & {$[3282.024-1381.302-1429.027]$} & {$[3499.620-574.505-1426.443]$} \\
12 & {$[25300-3500-2220]$} & {$[1302.215-1494.997-1425.853]$} & {$[1255.501-1298.326-1427.859]$} & {$[1507.215-954.028-1427.284]$} \\
13 & {$[21300-3500-2220]$} & {$[-664.617-1347.160-1419.009]$} & {$[-714.029-1192.874-1420.513]$} & {$[-434.865-1298.721-1421.633]$} \\
14 & {$[19300-3500-2220]$} & {$[-2639.386-1222.168-1411.597]$} & {$[-2691.028-1110.431-1412.373]$} & {$[-2378.904-1667.486-1415.462]$} \\
\hline
\end{tabular}

$$
\Delta q=\left(J_{q}^{T} J_{q}+n I\right)^{-1} J_{q}^{T} J_{l} \cdot \Delta l .
$$

where $n$ is a very small real number and $I$ is a unit matrix. The item $n I$ is an addition item to make sure the inverse of the matrix is not singular. In an iteration calculation process, the differential items $\delta q$ and $\delta l$ are written in a different form. The iteration calibration process is shown in Figure 6.

\section{Calibration Analysis and Docking Experiment}

4.1. Ground Datum Calibration Experiment. The ground datum calibration experiment process is shown in Figure 3. A laser tracker of Leica-AT960 is adopted to measure the ground datum points. The principle positions and the practical measurement values of the ground datum points are shown in Table 1 and Figure 7 (a). With the method proposed in Section 3.2, the second and third sets of practical measurement values should be transformed to the first measurement coordinate to minimize the measurement error. According to equations (1) (3), the transformed matrix from datum 2 to datum 1 is as follows:

$$
T_{d 2}^{d 1}=\left[\begin{array}{cccc}
0.99977 & 0.02153 & -0.00025 & 74.57361 \\
-0.02153 & 0.99977 & 0.00114 & -168.31363 \\
0.00028 & -0.00114 & 0.99999 & 0.36183 \\
0 & 0 & 0 & 1
\end{array}\right],
$$

and the transformed matrix from datum 3 to datum 1 is as follows:

$$
T_{d 3}^{d 1}=\left[\begin{array}{cccc}
0.96875 & 0.24805 & 0.00073 & 79.81523 \\
-0.24805 & 0.96875 & 0.00138 & -194.94554 \\
-0.00036 & -0.00152 & 0.99999 & 0.52415 \\
0 & 0 & 0 & 1
\end{array}\right] .
$$

The matched measurement datum points are shown in Figure 7(b). After being matched, the mean values of the datum points are calculated as the standard measurement datum to match the principle datum. The matching error rapidly decreases in the iteration process. After three iterations, the maximal distance error from the principle datum and the mean measurement datum was $1.91480496791221 e-11 \mathrm{~mm}$ (Figure 8). 


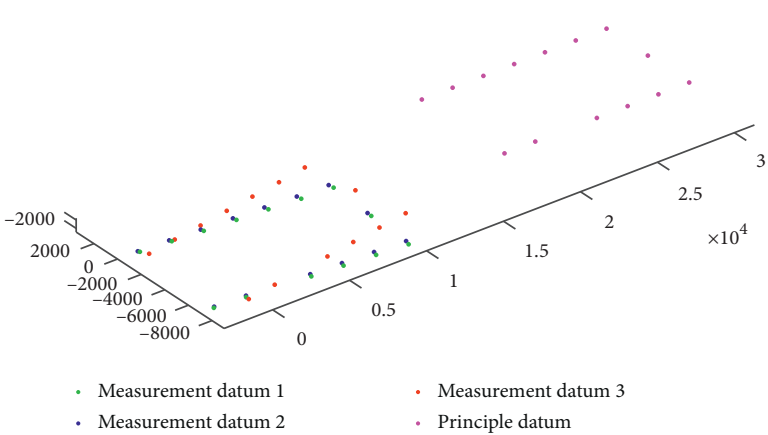

(a)

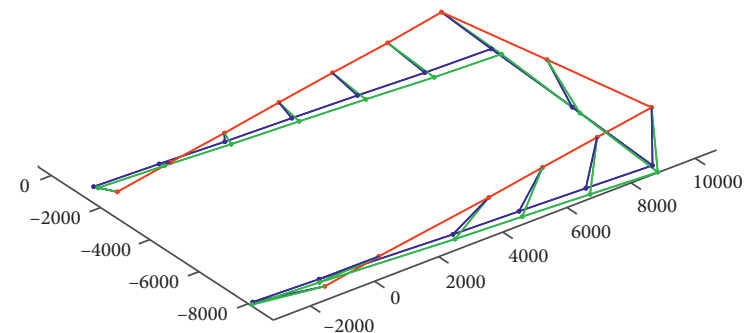

(b)

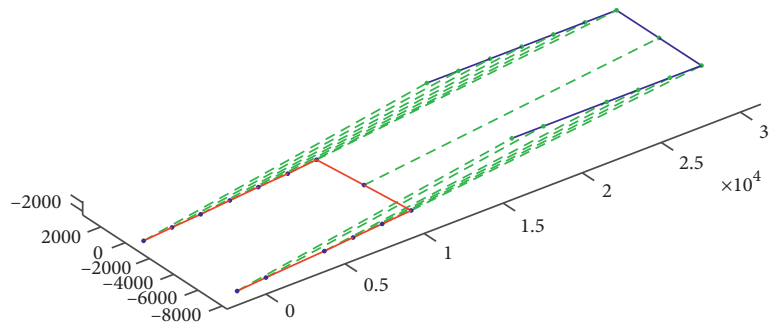

(c)

Figure 7: Ground datum point calibration process. (a) The principle datum and the practical measurement points (unit: $\mathrm{mm}$ ). The matching process of (b) the practical measurement points (unit: $\mathrm{mm}$ ) and (c) the mean points and the principle datum (unit: $\mathrm{mm}$ ).

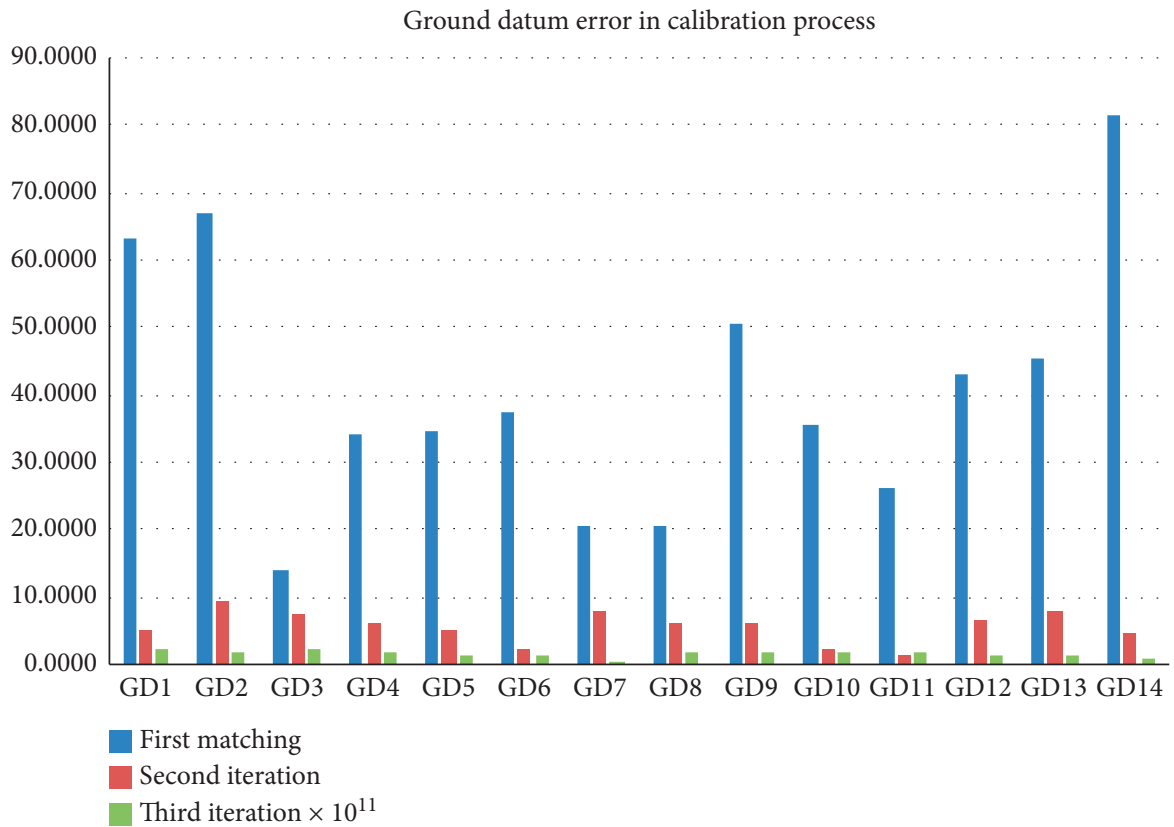

Figure 8: Ground datum point calibration error (unit: $\mathrm{mm}$ ).

4.2. Kinematic Calibration Experiment. The initial state of the positioners was set to approximate the ideal positions as designed in the numerical model and then fix the carrier on the positioners. Then when the parallel structure moves, the ideal geometrical parameters were used. As we know, the real geometric parameters cannot be absolutely the same with the designed model as the machining error and deformation were inevitable. But as the mounting holes of the carrier were machined by CNC machine tool, even though the carrier deforms, the real shape and designed shape would be very similar. The docking system can move with error but without mechanical stuck. Then, the calibration process can be carried out.

The calibration measurement process is shown in Figure 9. With the method proposed in Section 2, the airplane coordinate is conformed. And, in the same time, the relationship between the airplane coordinate and the measurement coordinate is decided. The poses and postures of 


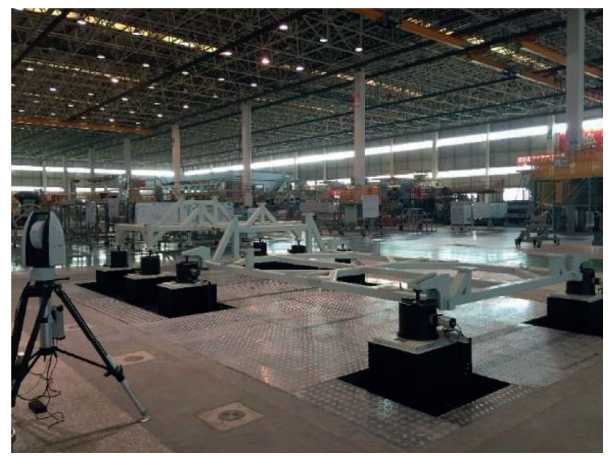

Figure 9: Position and posture measurement.

TABLE 2: Initial principle parameters and corresponding corrected values (unit: $\mathrm{mm}$ ).

\begin{tabular}{lcccc}
\hline \multirow{2}{*}{ Item } & Aft fuselage & \multicolumn{2}{c}{ Rear fuselage } \\
& Principle value & Actual value & Principle value & Actual value \\
\hline$P_{c}$ & $26181,0,-905$ & $26182.72,-1.23,-904.02$ & $26161,0,-905$ & $26160.56,-0.36,-904.78$ \\
$R_{c}^{w}$ & $0.00,0.00,0.00$ & $0.0076,0.0138,-0.1687$ & $0.00,0.00,0.00$ & $0.0135,-0.0268,0.2037$ \\
$v_{p 1}^{c}$ & $-5312,-1740,-585$ & $-5308.09,-1754.34,-589.12$ & $691,-1740,-585$ & $685.57,-1741.38,-586.97$ \\
$v_{p 2}^{c}$ & $-5312,1740,-585$ & $-5318.72,1725.73,-585.74$ & $691,1740,-585$ & $696.71,1739.13,-585.60$ \\
$v_{p 3}^{c}$ & $-668,-1740,-585$ & $-661.76,-1742.04,-586.42$ & $2238,-1740,-585$ & $2201.60,-1747.67,-586.74$ \\
$v_{p 4}^{c}$ & $-668,1740,-585$ & $-675.52,1737.66,-588.05$ & $2238,1740,-585$ & $2216.30,1731.83,-587.71$ \\
$d_{1}^{w}$ & $20869,-1740,-1490$ & $20869.35,-1739.85,-1492.09$ & $26852,-1740,-1490$ & $26852.59,-1739.15,-1491.84$ \\
$d_{2}^{w}$ & $20869,1740,-1490$ & $20868.96,1740.23,-1488.25$ & $26852,1740,-1490$ & $26851.36,1741.37,-1489.64$ \\
$d_{3}^{w}$ & $25513,-1740,-1490$ & $25515.69,-1741.24,-1490.51$ & $28399,-1740,-1490$ & $283663,-1740.05,-1490.90$ \\
$d_{4}^{w}$ & $25513,1740,-1490$ & $25512.18,1738.49,-1491.68$ & $28399,1740,-1490$ & $28370.96,1739.48,-1491.05$ \\
$\varepsilon_{p 1}^{w}$ & $0.00,0.00,0.00$ & $0.0235,0.0026,-0.1555$ & $0.00,0.00,0.00$ & $-0.0086,0.0233,0.1277$ \\
$\varepsilon_{p 2}^{w}$ & $0.00,0.00,0.00$ & $0.0050,0.0118,-0.1866$ & $0.00,0.00,0.00$ & $-0.0306,-0.0340,-0.2502$ \\
$\varepsilon_{p 3}^{w}$ & $0.00,0.00,0.00$ & $-0.0065,0.0164,-0.1983$ & $0.00,0.00,0.00$ & $-0.0077,-0.0375,-0.4158$ \\
$\varepsilon_{p 4}^{w}$ & $0.00,0.00,0.00$ & $-0.0019,0.0118,-0.1588$ & $0.00,0.00,0.00$ & $0.0348,-0.0157,-0.1820$ \\
\hline
\end{tabular}

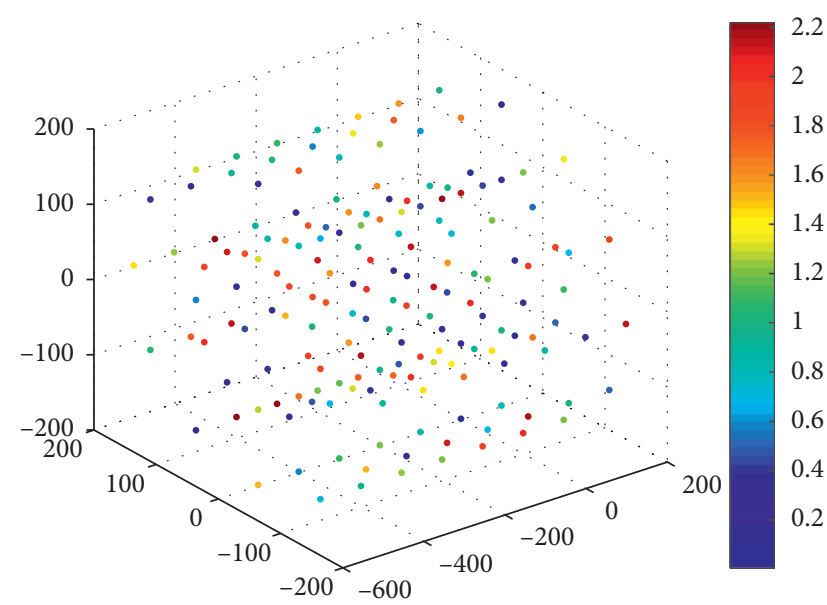

(a)

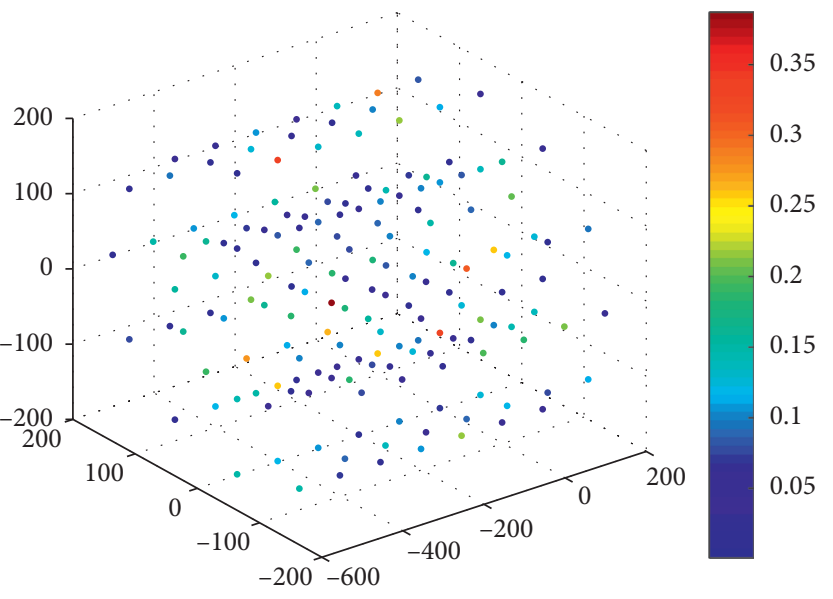

(b)

FIGURE 10: Distance error from the principle position to the actual position (unit: $\mathrm{mm}$ ). Position error (a) before calibration and (b) after calibration.

the carriers are changed with the motions of the positioners. A Leica930 tracker is used to measure the carrier coordinates. With the algorithm proposed in Section 3, 42 parameters of the docking system are calibrated. The initial principle values and the corrected value after calibration are shown in Table 2. In Table $2, \varepsilon_{p 1}^{w}, \varepsilon_{p 2}^{w}, \varepsilon_{p 3}^{w}$, and $\varepsilon_{p 4}^{w}$ are the corresponding Euler angles of the posture translation matrix from the four positioners to the airplane coordinate.

To prove the accuracy and validity of the calibrated parameters, the rear fuselage was taken as an example. From $-500 \mathrm{~mm}$ to $100 \mathrm{~mm}$ in the $X$ direction of the carrier coordinate, 16 points in every $Y Z$ plane with $100 \mathrm{~mm}$ interval 


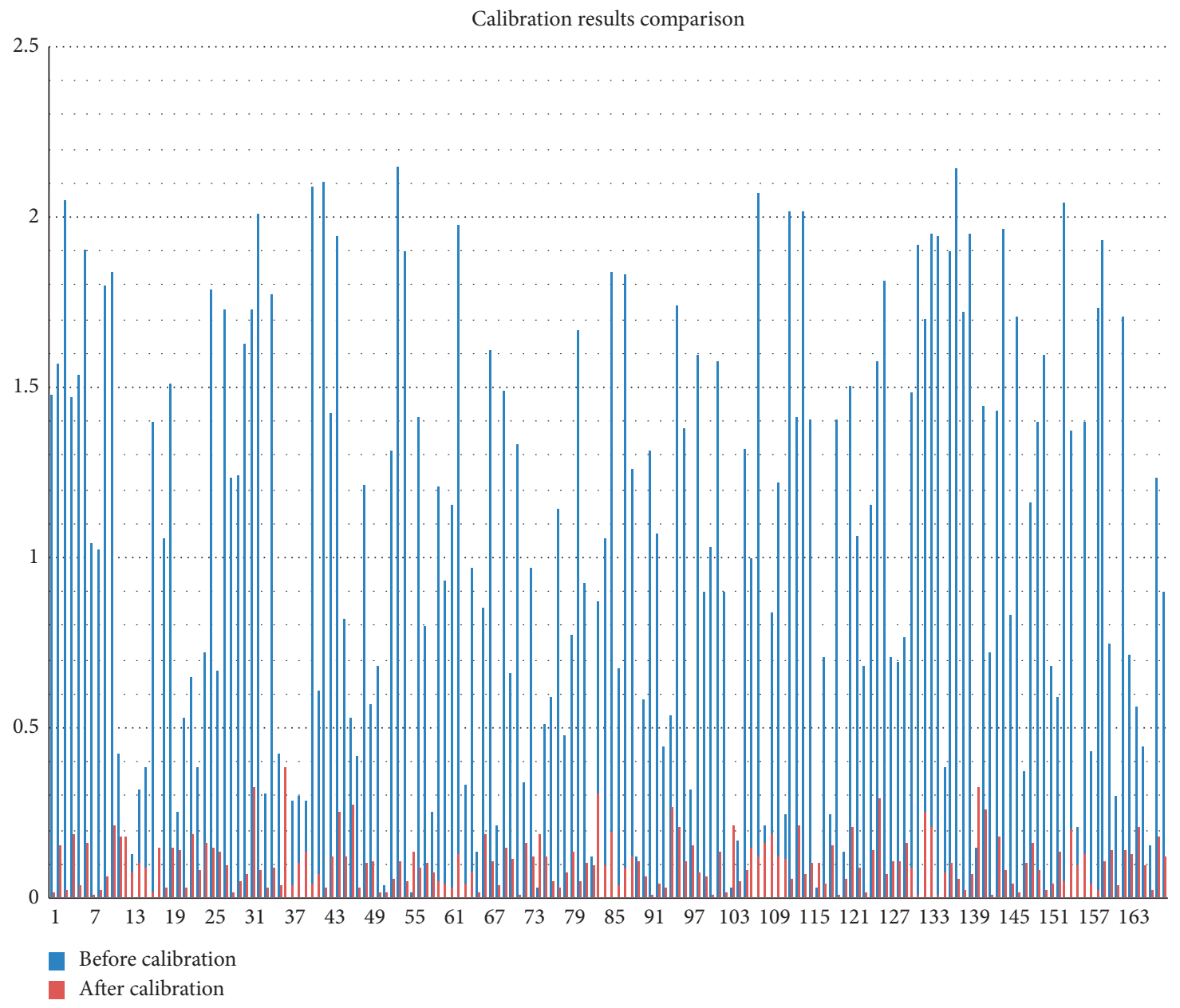

FIGURE 11: Contrast diagram of distance error (unit: mm).

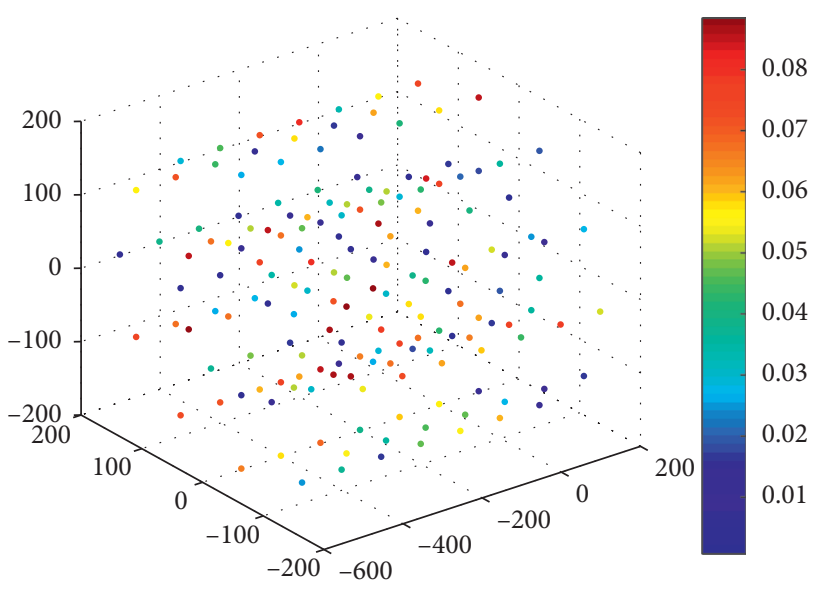

(a)

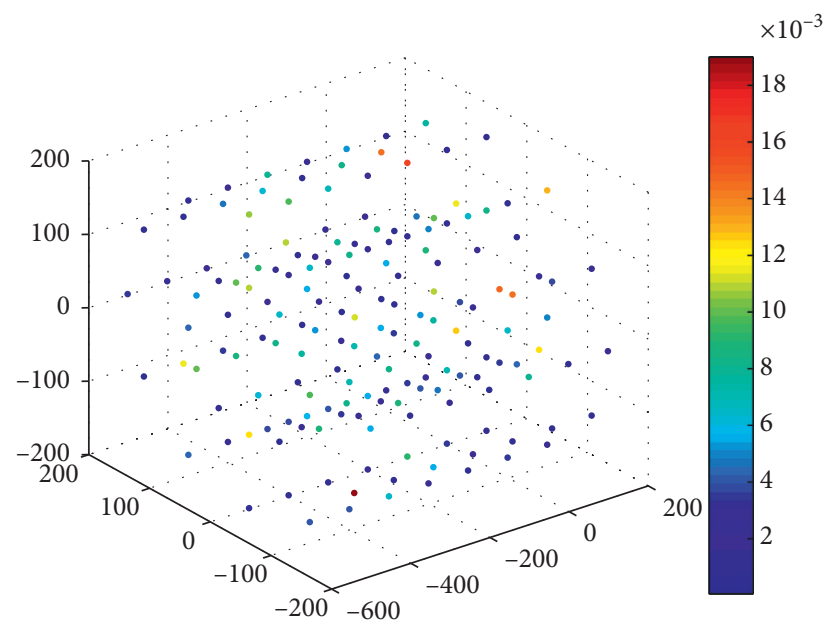

(b)

FIgURE 12: Angle error between the $X$ vector of the principle and actual coordinate (unit: degree). Angle error (a) before calibration and (b) after calibration.

distance were measured. The real measurement data were used to compare the two sets of pose and posture values which were calculated by the principle parameters and corrected parameters, respectively. For the $X$ axis of the coordinate points in the main direction of docking movement, the pointing accuracy error of the $X$ axis is used to 


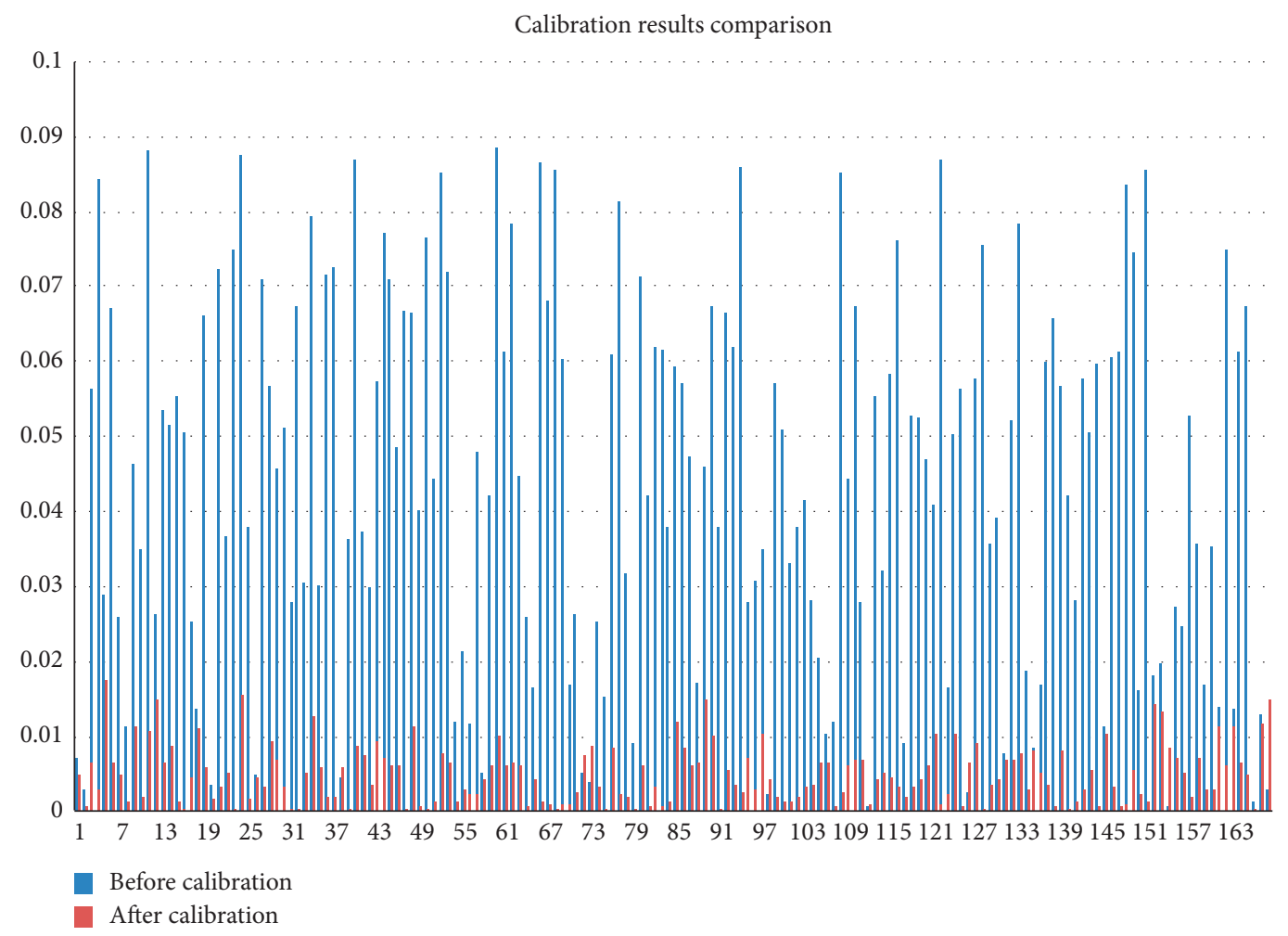

FIgURE 13: Contrast diagram of angle error (unit: degree).

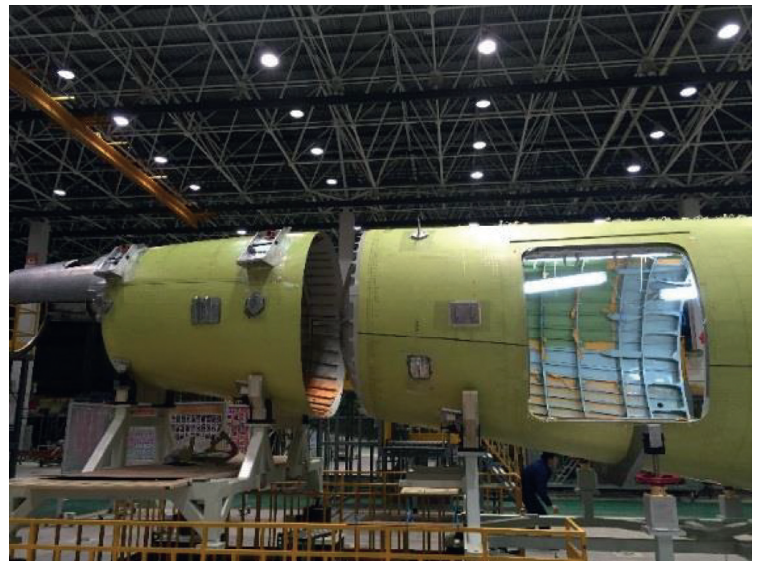

(a)

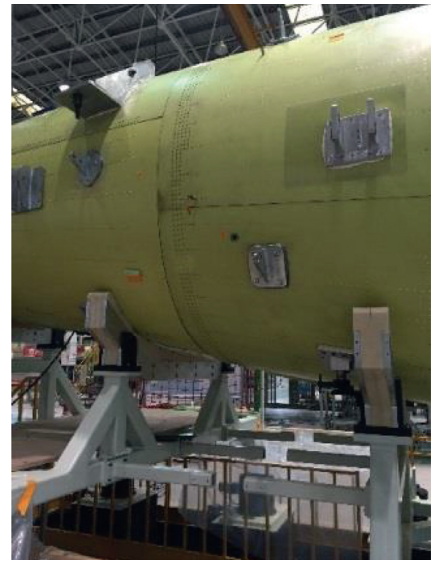

(b)

FIGURE 14: The automatic docking process of the first MA700 airplane.

assess the posture error. The position error comparison is shown in Figure 10. Before calibration, the maximal distance error between the principle positions calculated by the structure parameters and the measurement positions is $2.2 \mathrm{~mm}$ which decreases to 0.0035 after calibration. The position errors of corresponding points are shown in Figure 11.

At every measurement point, the pointing accuracy of the $X$ axis of the carrier coordinate was also calculated and compared which is shown in Figures 12 and 13. Before calibration, the maximum angle error was near 0.09 degree. However, the calibration algorithm reduced the angle error down to 0.018 degree. The angle error comparison at every measurement point is shown in Figure 13.

With the two calibration algorithms proposed in this paper, the rear and aft fuselages of the airplane can dock together very well as shown in Figure 14 . With the automatic docking system, the docking efficiency has been significantly improved from 2 days by manual work to just 5 minutes.

\section{Conclusion}

The double-position 4-PPPS aircraft docking system is a large distributed mechanism that has a mass of machining 
error, assembly error, and location error. The accuracy promotion of the docking system could improve the aircraft manufacturing accuracy, decrease the internal stress of the connection, and offer better guarantee of flight safety. And thus, two steps of accuracy improvement calibrations are proposed in the paper. The first calibration step promotes the accuracy of the relationship between the airplane coordinate and the measurement coordinate. An averaging iteration method is proposed to approach the real datum points. It is the basics of accurate measurement. And the second step promotes the accuracy of the mechanical structure parameters. That is to say, it improves the movement accuracy of the entire mechanism. An algorithm deduced by the derivative of the spatial pose transformation is proposed to calibrate up to 42 kinematic parameters. With the algorithms proposed, the maximum position error of the docking system deduced from $2.2 \mathrm{~mm}$ to $0.035 \mathrm{~mm}$ and the maximum pointing error deduced from 0.08 degree to 0.018 degree.

The real strokes of a positioner in its $X, Y$, and $Z$ directions are all near $1000 \mathrm{~mm}$. But as we know, the pose and posture error of two fuselages to be jointed together cannot be that big. When the carrier tilts too much, the deformation should be much larger than the value of the carrier is in its normal posture. The deformation is not a linear function jet. As the fuselages are all fixed by some location elements, the adjustments will be all around the initial state. After experiments, the real work range of $Y$ and $Z$ directions is set $\pm 200 \mathrm{~mm}$. At this range, the calibrated parameters are effective.

In fact, without the calibration process, the docking system cannot work well for the corresponding axes of the positioners are unparallel. They form large internal stress when the positioners move simultaneously. The algorithms proposed in this paper are effective and feasible. The next step of the research will go into the dynamics of the docking system.

\section{Data Availability}

The data used to support the findings of this study are included within the article.

\section{Conflicts of Interest}

The authors declare that they have no conflicts of interest.

\section{Acknowledgments}

The authors are grateful to the support provided by the project funded by the China Postdoctoral Science Foundation (2019M651145), State Key Laboratory of Robotics (2019-O21), and Natural Science Foundation of Liaoning Province (2019-ZD-0663).

\section{References}

[1] M. Boukattaya, M. Jallouli, and T. Damak, "On trajectory tracking control for nonholonomic mobile manipulators with dynamic uncertainties and external torque disturbances," Robotics and Autonomous Systems, vol. 60, no. 12, pp. 16401647, 2012.
[2] R. He, X. Li, T. Shi et al., "A kinematic calibration method based on the product of exponentials formula for serial robot using position measurements," Robotica, vol. 33, no. 6, pp. 1295-1313, 2014.

[3] F. Li, Q. Zeng, K. F. Ehmann, J. Cao, and T. Li, “A calibration method for overconstrained spatial translational parallel manipulators," Robotics and Computer-Integrated Manufacturing, vol. 57, pp. 241-254, 2019.

[4] Y. Jiang, T. Li, L. Wang, and F. Chen, "Improving tracking accuracy of a novel 3-DOF redundant planar parallel kinematic machine," Mechanism and Machine Theory, vol. 119, pp. 198-218, 2018.

[5] G. Ecorchard, R. Neugebauer, and P. Maurine, "Elasto-geometrical modeling and calibration of redundantly actuated PKMs," Mechanism and Machine Theory, vol. 45, no. 5, pp. 795-810, 2010.

[6] Z. Q. Ma, S. G. Li, H. W. Xing, and H. Xiang, "Analytic forward solution for 3-PPPS parallel wing posture adjustment mechanism," Computer Integrated Manufacturing System, vol. 21, no. 2, pp. 449-454, 2015.

[7] Z. Q. Ma, S. G. Li, H. W. Xing, and H. Xiang, "Kinematic calibration of 3-PPPS parallel wing posture adjustment mechanism," Computer Integrated Manufacturing System, vol. 21, no. 9, pp. 2378-2383, 2015.

[8] L. Y. He, Q. C. Li, X. B. Zhu, and C. Y. Wu, "Kinematic calibration of a three degrees-of-freedom parallel manipulator with a laser tracker," Journal of Dynamic Systems Measurement and Control-Transactions of the ASME, vol. 141, no. 3, Article ID 031009, 2019.

[9] H. Tian, Z. Dong, F. W. Yin, W. J. Tian, and G. C. Derk, "Kinematic calibration of a 6-DOF hybrid robot by considering muticollinearity in the identification Jacobian," Mechanism and Machine Theory, vol. 131, pp. 371-384, 2019.

[10] G. Chen, L. Kong, Q. Li, H. Wang, and Z. Lin, "Complete minimal and continuous error models for the kinematic calibration of parallel manipulators based on POE formula," Mechanism and Machine Theory, vol. 121, pp. 844-856, 2018.

[11] K. Großmann and B. Kauschinger, "Eccentric universal joints for parallel kinematic machine tools: variants and kinematic transformations," Product Engineering, vol. 6, no. 4-5, pp. 521-529, 2012.

[12] C. Han, Y. Yu, Z. Xu, X. Wang, P. Yu, and X. Zhou, "Complete kinematic calibration of a 6-RRRPRR parallel kinematic machine based on the optimal measurement configrations," Proceedings of the Institution of Mechanical Engineers, Part C: Journal of Mechanical Engineering Science, vol. 234, no. 1, pp. 121-136, 2019.

[13] D. Y. Yu, D. C. Cong, and J. W. Han, "Parallel robots pose accuracy compensation using artificial neural networks," in Proceedings of the 4th Interational Conference on Machine Learning and Cybernetics, pp. 3194-3198, Guangzhou, China, August 2005.

[14] D. Zhang, G. Zhang, and L. Li, "Calibration of a six-axis parallel manipulator based on BP neural network," Industrial Robot: The International Journal of Robotics Research and Application, vol. 46, no. 5, pp. 692-698, 2019.

[15] C. Fan, G. L. Zhao, J. Zhao et al., "Calibration of a parallel mechanism in a serial-parallel polishing machine tool based on genetic algorithm," International Journal of Advanced Manufacturing Technology, vol. 81, no. 1-4, pp. 27-37, 2015.

[16] R. Hamid and H. Heikki, "A novel haptic interface and universal control strategy for international thermonuclear experimental reactor welding/machining assembly robot," 
Robotics and Computer Integrated Manufacturing, vol. 57, pp. 255-270, 2019.

[17] R. Qi, W. Zhou, and W. Tiejun, "Trajectory evaluation for manipulators with motion and sensor uncertainties," Industrial Robot: An International Journal, vol. 44, no. 5, pp. 658-670, 2017.

[18] Z. Jin, C. Yu, J. Li, and Y. Ke, "Configuration analysis of the ERS points in large-volume metrology system," Sensors, vol. 15, no. 9, pp. 24397-24408, 2015.

[19] R. L. Qi, W. Zhang, Y. Z. Tian, and L. Xiao, "High order curvature and torsion continuous trajectory planning method for space flight robot," in Proceedings of the 7th International Conference on Intelligent Human-Machine Systems and Cybernetics, pp. 60-63, Hangzhou, China, August 2015.

[20] Z. H. Wang, H. Xu, G. D. Chen, R. C. Sun, and L. N. Sun, "A distance error based industrial robot kinematic calibration method," Industrial Robot: An International Journal, vol. 41, no. 5, pp. 439-446, 2014. 